\title{
Optical contamination control in the Advanced LIGO ultra-high vacuum system
}

\author{
Margot H. Phelps ${ }^{\mathrm{a}}$, Kaitlin E. Gushwa ${ }^{\mathrm{a}}$, and Calum I. Torrie ${ }^{\mathrm{a}, \mathrm{b}}$ \\ ${ }^{a}$ LIGO Laboratory, California Institute of Technology, 1200 E. California Blvd., Pasadena, CA \\ USA 91125; 'bUPA, School of Physics and Astronomy, Kelvin Building, University of Glasgow, \\ Glasgow G12 8QQ UK
}

\begin{abstract}
Fused silica optics in the Advanced Laser Interferometer Gravitational-Wave Observatory (LIGO) detectors are extremely sensitive to optical scattering and absorption losses induced by both particulate and hydrocarbon contamination. At full power, the optical surfaces are illuminated with up to $200 \mathrm{~kW} / \mathrm{cm}^{2}$. Additionally, the round-trip test mass cavity loss budget is limited to $70 \mathrm{ppm}$ total from all sources. Even low-level contaminants can result in laser damage to optics during the operation the interferometers, and/or the unacceptable reduction of overall detector sensitivity. These risks are mitigated by a two-pronged approach: quantifying contamination sources and the extent of contamination, then reducing sources and cleaning optics in-situ. As a result of these ongoing efforts, we now have a better understanding of what the contamination levels and sources are, and have made significant improvements to methods of controlling contamination, thus protecting the optics from losses and laser damage in the Advanced LIGO Interferometers.
\end{abstract}

Keywords: LIGO, fused silica optics, absorption, scatter, contamination control, cleanliness, ultra-high vacuum system, mitigation tools

\section{INTRODUCTION}

LIGO consists of two ground-based Michelson interferometers with Fabry-Perot arm cavities, operated in unison with the aim of directly detecting gravitational waves (GWs). These detectors, located in Livingston, LA and Hanford, WA, are comprised of vacuum chambers that house individual suspended optical components with connecting beamtubes that form an L shape, as shown below.

A laser is split into two beams that travel down the $4 \mathrm{~km}$ long arms at the same time, where they resonate in the two

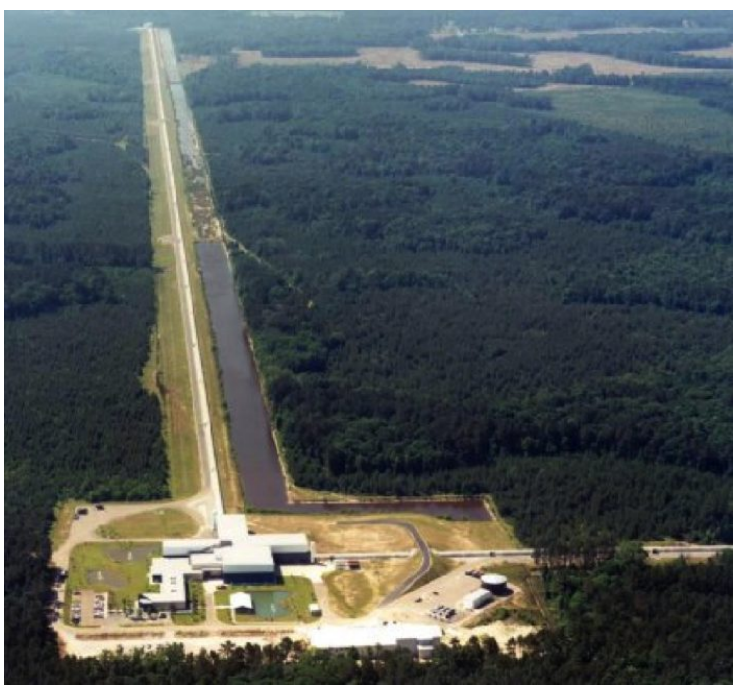

Figure 1. LIGO Livingston observatory.

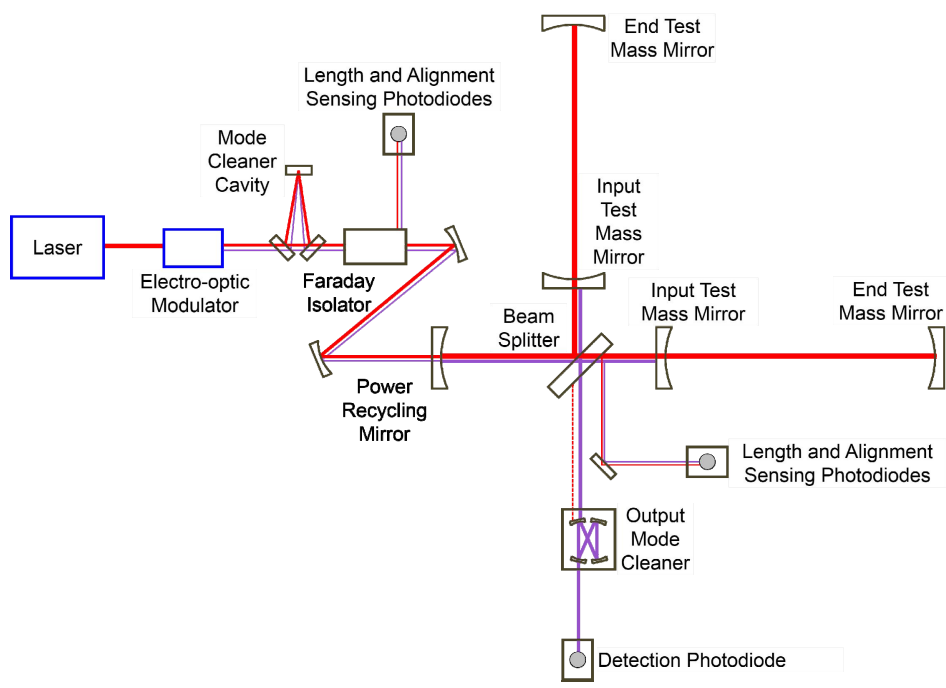

Figure 2. Basic optical layout of the LIGO interferometer.

Laser-Induced Damage in Optical Materials: 2013, edited by Gregory J. Exarhos, Vitaly E. Gruzdev, Joseph A. Menapace, Detlev Ristau, MJ Soileau, Proc. of SPIE Vol. 8885, 88852E - (c) 2013 SPIE - CCC code: 0277-786X/13/\$18 - doi: 10.1117/12.2047327 
Fabry-Perot cavities before returning to the beam splitter. If the light travels identical distances along each arm, it will fully destructively interfere when recombined. On the other hand, if there is any difference between the lengths of the beam paths, some of the light will be detected by a photodetector. A GW passing through the interferometer would shorten or lengthen one of the arms with respect to the other, resulting in a light signal and GW detection.

Advanced LIGO is an upgrade of the Initial LIGO detectors, an endeavor to increase the observatory's sensitivity to a level where GW signals are routinely detected. The upgrades include, but are not limited to, new optics and higher power lasers ${ }^{1}$. Attaining routine detections requires being incredibly sensitive to changes in distance, on the order of $10^{-19} \mathrm{~m}$ across the $4 \mathrm{~km}$ arms. There is a long list of noise sources that can potentially reduce the sensitivity of the system, and all of the interferometer components were designed with this in mind. LIGO's fused silica optics are designed to have very low intrinsic absorption and scatter; however, they are also extremely sensitive to scatter and absorption loss induced by particulate and hydrocarbon contamination. Because optical performance is paramount to the success of the interferometers, reducing contamination in the ultra-high vacuum (UHV) system is critical.

\section{REQUIREMENTS}

\subsection{Optical requirements}

The interferometers' core optics are large fused silica optics that are hung from multi-stage pendulum suspensions which provide isolation from seismic motion. The optical coatings were designed to have absorption less than $1 \mathrm{ppm}$ and scatter less than $1 \mathrm{ppm}$ per cavity mirror surface. In addition to the scatter and absorption requirements per surface, the losses of each subsystem of the interferometer have to be considered, especially of the Fabry-Perot cavity itself.

The Fabry-Perot cavity is made up of an input test mass (ITM) and an end test mass (ETM). The allowable round-trip loss for each cavity, called the cavity loss budget, is $70 \mathrm{ppm}$. The requirements for absorption of each cavity surface are also strict at $0.5 \mathrm{ppm}$ per surface, with $0.1 \mathrm{ppm}$ set aside for the contamination budget. One single $30 \mu \mathrm{m}$ particle in the beam path accounts for $0.1 \mathrm{ppm}$, and to date several particles of this size have been found over most optical surfaces ${ }^{2}$.

Keeping contamination-induced optical absorption and scatter low is important to meet LIGO's design sensitivity, and also to prevent laser damage to optical surfaces. Portions of the LIGO system (the input mode cleaner mirrors) see peak laser intensities of $200 \mathrm{~kW} / \mathrm{cm}^{2}$. Particulate or hydrocarbon contamination on optical surfaces in that beam path could result in laser damage that would negatively impact interferometer operations. To mimic the potential danger to an Advanced LIGO mode cleaner optic, a witness sample optic was left in chamber to gather contamination before being sent to Caltech, where it was irradiated with a laser of the same wavelength and similar power to the one that would be used in the mode cleaner. Figure 3 is an image taken on a Scanning Electron Microscope (SEM) of the site of a

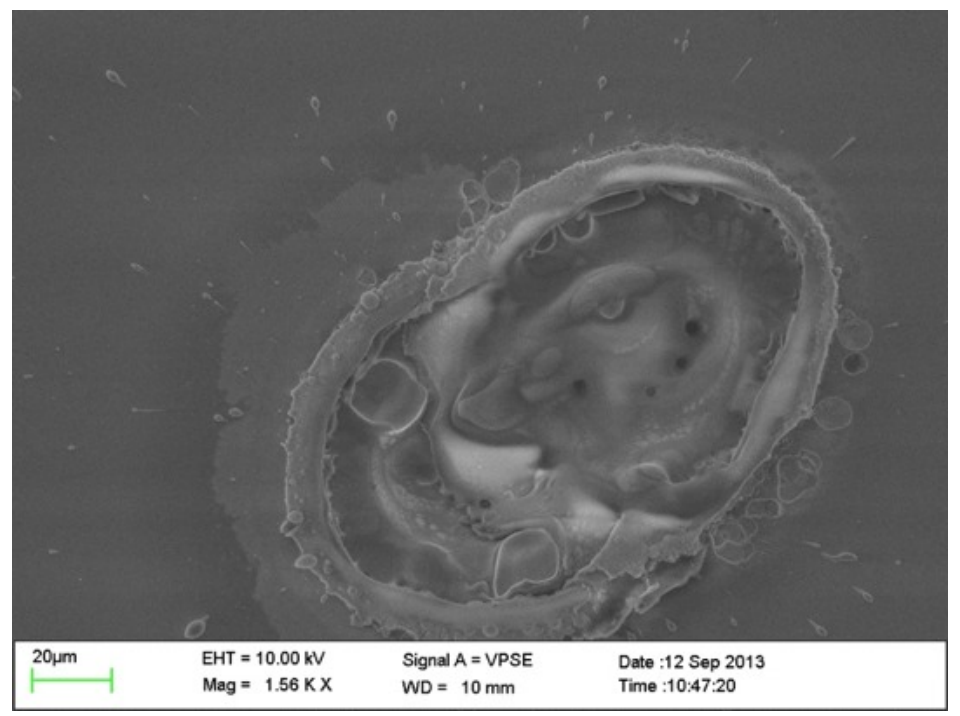

Figure 3. SEM image of an intentional laser damage test mimicking what the LIGO laser would do to a contaminant on the surface of a mode cleaner during high power operation. 
particulate contaminant, and highlights the result of the intentional laser damage test.

\subsection{Cleanliness requirements}

If a component will be exposed to the interferometer vacuum system while under vacuum, it is defined as Class A. An installation tool that comes into contact with Class A parts or vacuum equipment that will be exposed to vacuum is called a Class $\mathrm{B}^{3}$. For example, a suspension assembly is Class A, while the Allen key used in its assembly is Class B. Class A parts consist solely of the materials found on the LIGO Vacuum Compatible Materials List ${ }^{4}$, all of which passed a rigorous qualification procedure. This list was made primarily to limit or remove materials that would outgas in vacuum, similar to NASA outgassing specifications ${ }^{5}$.

Once a Class A part is manufactured with an acceptable material, it then goes through a stringent clean and vacuum bake process that includes passing a Residual Gas Analyzer (RGA) scan, which is carried out using a small mass spectrometer attached to the vacuum bake ovens ${ }^{6}$. Class B parts follow the same cleaning procedures and bake temperatures as Class A parts of the same material, but the bake is performed in air for 24 hours. Only if a part passes all these steps is it allowed into the chamber.

The cleanliness of work areas is also monitored to prevent contamination. Cleanrooms are defined by cleanliness class, which refers to the quantity and size of particles permitted per volume of air in the room. The LIGO vacuum chambers operate in an ISO 5 (Class 100) environment, and air quality is frequently checked with handheld particle counters ${ }^{6}$. Similarly, surfaces in cleanrooms and thin-films are defined by a cleanliness level, which is the particle size distribution criteria per area specified by IEST-STD-CC1246D ${ }^{7}$ with the following equation:

$$
N=0.926\left(\log ^{2} L-\log ^{2} x\right)
$$

Where $N$ is the number of particles per $0.1 \mathrm{~m}^{2}$ of surface, 0.926 is the absolute value of the slope of the line, $x$ is the particle size $(\mu \mathrm{m})$, and $L$ is the cleanliness level. These levels, which will be referred to in this paper as Particulate Cleanliness Levels (PCLs), are named for the largest particle found per specified area. For example, a single $30 \mu \mathrm{m}$ diameter particle on a $0.1 \mu \mathrm{m} 2$ surface corresponds to a PCL 30. The PCL provides a quantitative and practical way to track particulate levels on surfaces during assembly and installation work. LIGO's design sensitivity correlates to a goal PCL of 65 for all vertical suspended optical surfaces ${ }^{2}$.

\section{DIAGNOSTIC TOOLS AND TECHNIQUES}

Despite strict cleanliness protocols in LIGO cleanrooms, visible contamination has been found on surfaces in assembly areas and in chamber at both observatories. Since the particulates were not detected by cleanroom particle counters, a new set of tools was implemented to help quantify the extent of contamination and identify its sources.

\subsection{Particle size distribution analysis}

IEST-STD-CC1246 standardizes the criteria for PCLs, but does not fully define the method for measuring them. LIGO has employed short and long-term analysis techniques to calculate and monitor changes in PCLs of surfaces in the chambers.

\subsubsection{Particle Cleanliness Validation System}

The National Ignition Facility (NIF) developed a dry surface swiping technique to collect particulates on a membrane filter for analysis under a microscope ${ }^{8}$. Following discussions with NIF, LIGO created a Particle Cleanliness Validation System (PCVS) ${ }^{9}$ based on NIF's design to take dust samples from tables and instruments inside cleanrooms and vacuum chambers. A filter is clipped onto the swipe tool, placed flat on a horizontal surface, and dragged slowly across the surface by holding the swipe tool at a 45 degree angle and applying light vertical pressure. A notable difference in the LIGO procedure is holding the trailing edge of the filter to the swipe tool body with a finger to constrain the area used for sample collection, as shown in Figure 5. The filter is then placed into a clean, clear, protective case for particle counting with a microscope. 


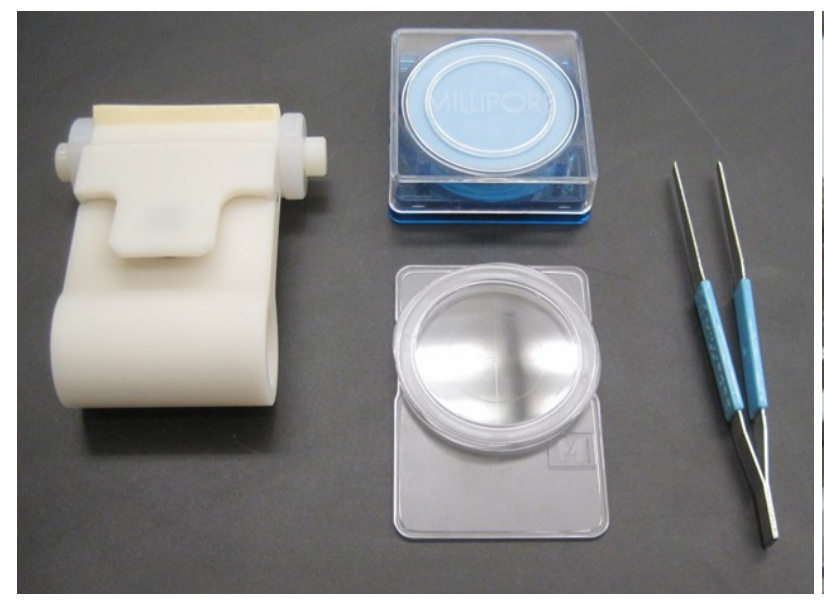

Figure 4. Swipe tool, polycarbonate membrane filters, PetriSlide, and filter forceps used for sample collection.

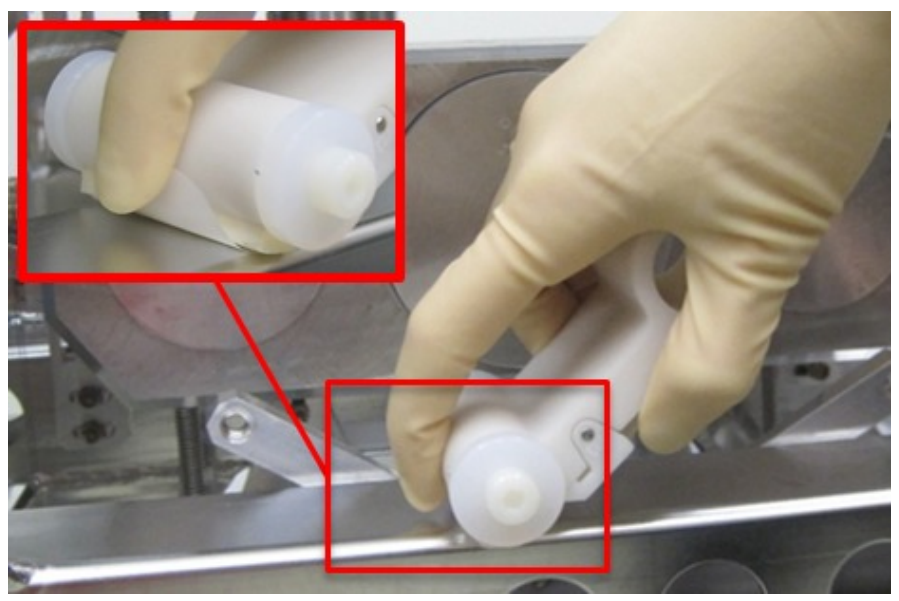

Figure 5. Swipe tool in action, Holding the free end of the filter concentrates particles to a smaller section of the filter.

NIF uses custom rectangular cellulose membrane filters and swipe cassette coves. To reduce costs, LIGO uses off-theshelf circular, polycarbonate membrane filters ${ }^{*}$ and transparent PetriSlides ${ }^{\dagger}$. An advantage of the smooth, semitransparent polycarbonate material is the ability to highlight both light and dark particles.

LIGO developed a unique image analysis procedure using a modified AmScope stereomicroscope, X-Y stage, digital camera, and ToupView software ${ }^{10}$. Video frames of the sample at $10 \mathrm{X}$ magnification are captured in columns and stitched into a single color image, which is then cropped to roughly $47 \mathrm{~mm} \times 8 \mathrm{~mm}$ to remove the edges of the filter. The disadvantage of circular filter compared to a rectangular one, is more of the sample must be cropped. Holding the free end of the filter to concentrate the collected particles to a smaller band minimizes the amount of sample excluded from analysis. The cropped image is then processed to enhance the light and dark particles, converted to binary, and the perimeter and area of each particle is computed.

These data are exported into a spreadsheet that calculates the PCL per IEST-STD-CC1246, and tracks these levels for given areas over time. LIGO campus labs and both observatories have identical PCVS. This process can quickly be performed on site, giving install teams a fast method of assessing the condition of the chamber they are working in, and complementing the use of a particle counter for cleanroom air.

\subsubsection{Witness wafers}

When it comes to measuring the long-term level of surface contamination in the vacuum chambers, 4 inch silicon wafers have been and continue to be the robust tool of choice. Clean wafers are set horizontally and vertically in chamber near optical surfaces, then collect contaminants through installation work and the pump down/vent of chambers. After they have seen a significant amount of installation work, they are removed and sent to Caltech for analysis.

At Caltech, a dark field microscope with an automated X-Y stage is used for scanning and counting the wafers. The center $2 \mathrm{~cm} \times 2 \mathrm{~cm}$ portion of each wafer is scanned, and the total count converted into a PCL. This approach provides a way to determine the cumulative particle distribution of each chamber in the system, as well as the ability to track those levels over time. Tracking them over time is key, because it allows us to correlate changes in PCL to different installation activities, and show which cleaning approaches are most effective. It also measures progress towards the goal PCL of 65 for vertical optical surfaces.

The installation and test schedule usually requires LIGO chambers to be open to air for a few months for install, then closed and pumped down to vacuum for testing, only to re-open later and have the cycle start again. The wafers are put in chamber at the start of this natural cycle and removed at the end of it, to standardize the amount of time/install work each wafer sees.

\footnotetext{
${ }^{*}$ EMD Millipore, P/N ATTP04700, www.millipore.com, (800) 646-5476

${ }^{\dagger}$ EMD Millipore, P/N PD1504700, www.millipore.com, (800) 646-5476
} 


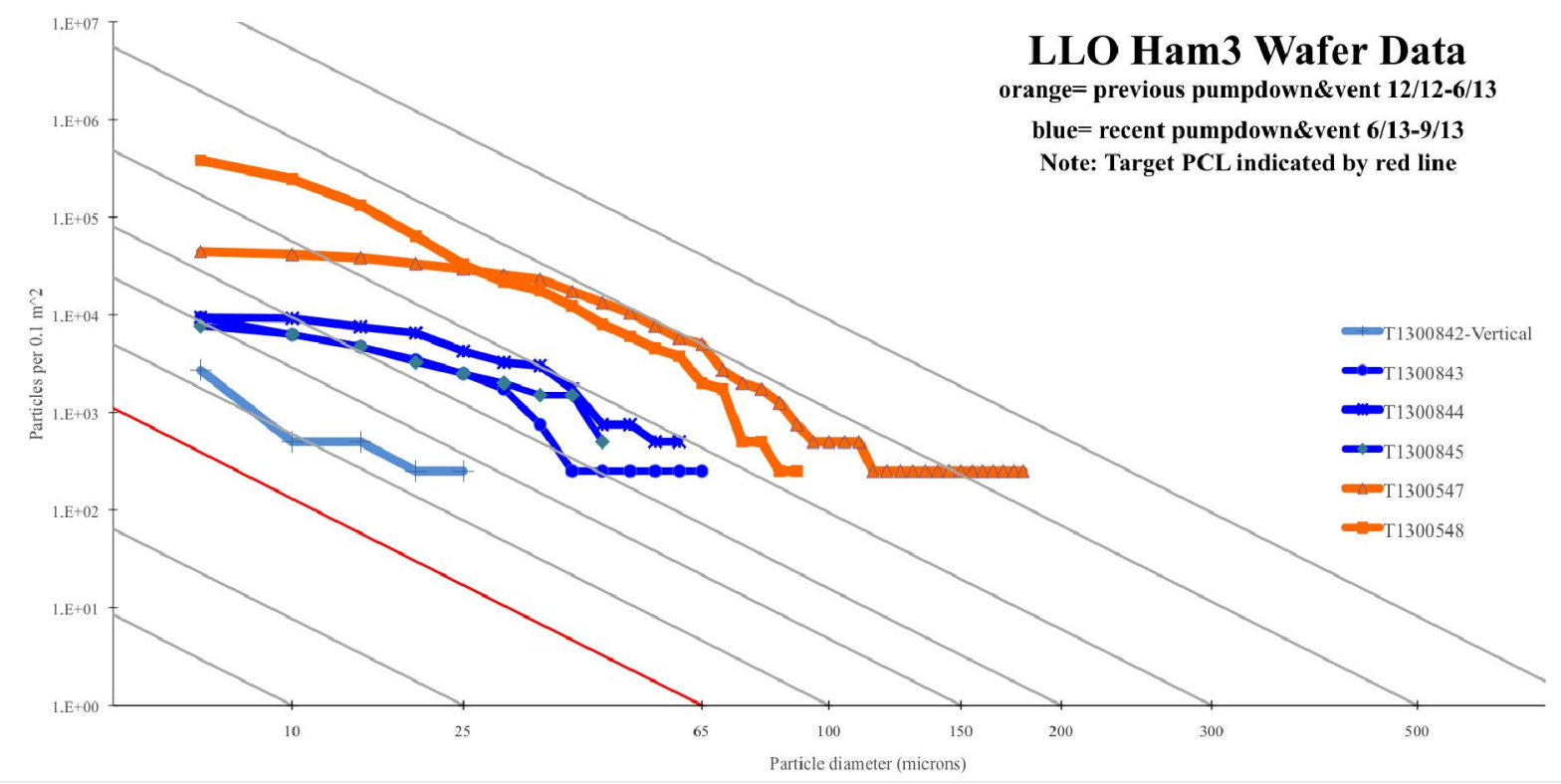

Figure 6. LIGO Livingston Observatory wafer counting data.

Figure 6 shows progress towards the goal PCL of 65 for one of our busiest chambers. The diagonal lines indicate the cleanliness levels as defined by IEST-STD-CC1246D, with LIGO's goal of PCL 65 indicated by the red line. One type of LIGO vacuum chamber is called a Horizontal Access Module (HAM). The data above is from our HAM3 chamber.

The orange datasets are counting data gathered in chamber during period of installation work then pump down and vent during 12/2012 through 6/2013. The blue trend lines are counting data gathered in chamber during a period of installation work followed by a pump-down and vent in the interval 6/2013 through 9/2013. All wafers were placed horizontally in HAM3 with the exception of T1300842, which was placed vertically.

Figure 6 shows PCLs in HAM3 that were as high as 590 in 12/2012 are now down in the 150-250 range as of pumpdown in $9 / 2013$. This decrease in particulate counts indicates a trend over time that is headed in the right direction towards our goal PCL of 65, from 12/2012 to now. This data is encouraging as it also shows fewer large particulates found in the more recent datasets, as well as a lower count of small particles.

\subsection{Chemical and molecular analysis}

Knowing the level of contamination present in the interferometers is only half the battle. Identifying the makeup of the particulates is the key to understanding where the debris came from and developing techniques to best combat the problem.

\subsection{1 "FBI samples"}

A commercially available adhesive tool is used to remove particles from a variety of surfaces for in-house examination at Caltech with a SEM. Known internally as an FBI sample due to its use in the forensic examination of gunshot residue, the tool consists of a $12 \mathrm{~mm}$ diameter ultra-smooth carbon adhesive tab stuck on a SEM specimen mount, which is inserted into a plastic base and then capped with a protective plastic tube. These sticky tabs are very effective at collecting particulates from a variety of surfaces (excluding optical surfaces). Any deposited adhesive is easily removed from sampled areas with Spectra-grade isopropyl soaked wipes. Tested areas were analyzed for residue by FTIR analysis, which passed, i.e. no detectable trace of residue was left ${ }^{11}$. Given the ease of use and low sampling costs, the tool has been popular. A growing library of samples has been collected and identified.

\footnotetext{
${ }^{\ddagger}$ Electron Microscopy Sciences, P/N 77827-12, www.ensdiasum.com, (215) 412-8400
} 


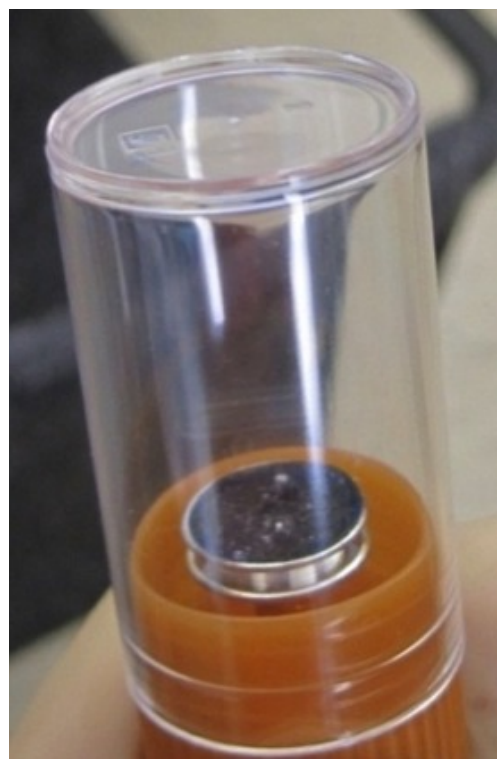

Figure 7. FBI sample tool.

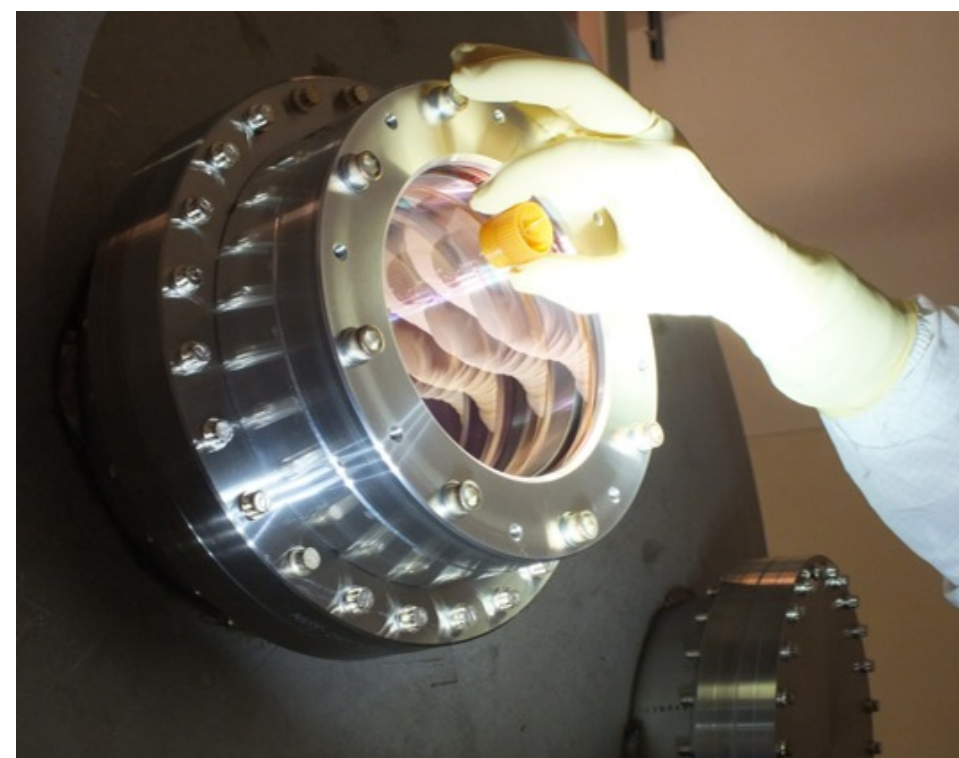

Figure 8. Collection of an FBI sample from a chamber viewport.

FBI samples were taken of "known suspects," materials commonly found in LIGO cleanrooms for comparison against "criminals," samples collected at the observatories. One of the most surprising discoveries came during the SEM analysis of a "known suspect" - cleanroom gloves. A fresh pair of gloves straight out of the packaging was donned, and one finger was lightly pressed onto an FBI sample. The density of particles from these supposedly clean gloves was very high.

Figure 10 summarizes the materials found in the Advanced LIGO vacuum systems so far. The most common specimens were metals, which is not surprising considering that most of the assemblies and the chambers themselves are metal. There was also a high amount of glove residue and fibers from supposedly clean garb, wipes, and glove liners. This result has forced LIGO to re-evaluate the use of these "clean" materials, as discussed in further detail in the Conclusions section. The total quantity of biological specimens is misleadingly low. It is important to note that, with the exception of hair, biological samples tend to be very small and are often overlooked. There is a tendency to focus analysis on larger particulates ( $20 \mu \mathrm{m}$ and greater) because they are easier to find and easier to get good readings from. It is difficult for the human eye and SEM to focus on very small object. There is also a tendency to collect samples of particulates that are large or out of the ordinary.

From the FBI samples, it is apparent that there is no smoking gun, i.e. no single source of contamination. Contamination is generated during assembly and installation activities performed by people - walking into a cleanroom, turning a screw, touching parts with gloved hands, and wiping down a work station are just a few examples.
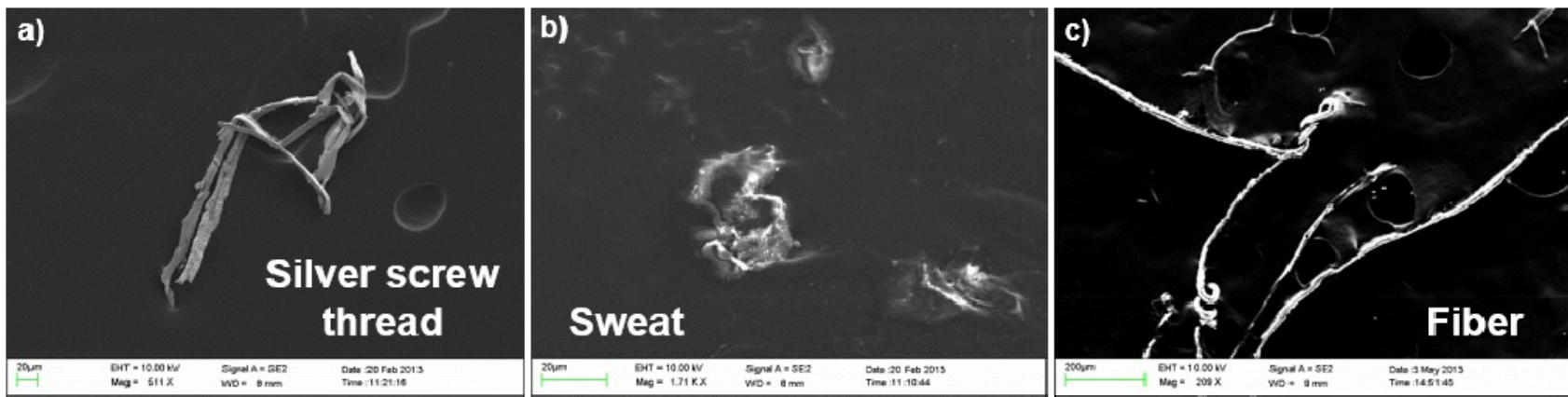

Figure 9. SEM images of FBI samples of a) a silver screw thread, b) dried sweat, and c) a fiber. All of these specimen were collected inside the vacuum system. 


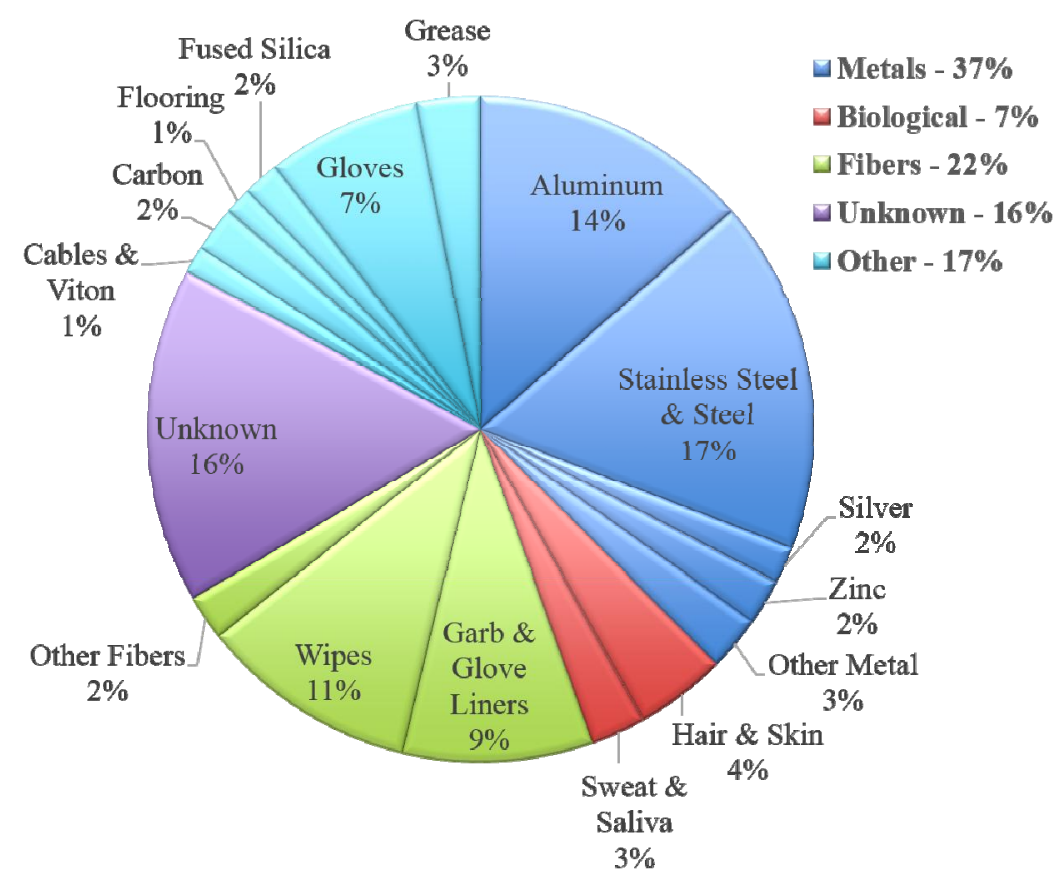

Figure 10. Distribution of specimens collected with FBI sample tool in vacuum chambers

\subsubsection{Witness wafers molecular analysis}

The SEM provided accessible on-campus means to get elemental data; however analyzing the molecular makeup of sources had to be done by outside vendors. Contamination was gathered from LIGO vacuum chambers on witness sample optics and silicon witness wafers. The samples were sent to two different vendors (referred to here as Vendor A and Vendor B). They ascertained the molecular makeup of the contamination samples using the following techniques:

Micro-Raman spectroscopy: This setup uses a $532 \mathrm{~nm}$ laser to excite a sample, then analyze its molecular makeup with a Raman microprobe. Vendor A used this to analyze LIGO samples.

Micro-Infrared spectroscopy: The material was analyzed using Fourier Transform Infrared Spectroscopy (FTIR) with a microscope attachment. This system was a Bruker Vertex 70v using a Hyperion microscope. This method is usually used in conjunction with Micro-Raman spectroscopy. Both Vendor A and B used this form of analysis.

Time-of-Flight Secondary Ion Mass Spectrometry (ToF-SIMS): A pulsed ion beam removes molecules from outer sample layer, and accelerates them into a flight tube. Their exact time-of-flight through the tube is recorded and a mass is determined from its duration. Vendor B used this to analyze LIGO samples.

Table 1. Summary of vendor molecular analysis.

\begin{tabular}{|l|l|l|}
\hline \multicolumn{1}{|c|}{ Method } & \multicolumn{1}{|c|}{ Sample } & \multicolumn{1}{c|}{ Findings } \\
\hline Micro-Raman Spectroscopy & Silicon wafer & Skin dander, silicates (dirt), cotton fibers \\
\hline $\begin{array}{l}\text { Micro-Infrared Spectroscopy } \\
\text { (FTIR) }\end{array}$ & Silicon wafer & $\begin{array}{l}\text { Skin dander, polyethers, silicones, organic acids } \\
\text { (possible lubricants) }\end{array}$ \\
\hline $\begin{array}{l}\text { Micro-Infrared Spectroscopy } \\
\text { (FTIR) }\end{array}$ & Silicon wafer & Skin dander, silicates (dirt), cotton fibers \\
\hline ToF-SIMS & Witness sample optic & $\begin{array}{l}\text { K, Cl, Br hydrocarbons and polydimethylsiloxane } \\
\text { (PDMS, possibly pump oil) }\end{array}$ \\
\hline
\end{tabular}


See Table 1 for the molecular analysis results. None of these approaches can be used to detect metal, which was easily identified through elemental analysis. Most common sources found from this approach were silicates, probably from dirt tracked in by shoes, and also skin dander, cotton fibers, and possible lubricants.

\subsubsection{Witness sample optics}

In addition to the silicon wafers that are placed in chamber, there are also 1 inch optics that are placed vertically and horizontally near optical surfaces. Ion Beam Sputtered (IBS) coated $1064 \mathrm{~nm}$ optics were selected to serve as witness samples for all the important in-chamber surfaces. Since they are basically small versions of the large real IBS coated $1064 \mathrm{~nm}$ HR/AR optics, they can be removed and measured for scatter and absorption, which would be analogous to doing a measurement on the real optical surfaces. This is a good way to assess risk to optical surfaces, especially in the form of hydrocarbon induced absorption or contaminants that lead to laser damage.

\section{MITIGATION TOOLS}

Extensive sample collection and testing has highlighted the magnitude and complexity of the contamination problem. Mitigation and cleanup efforts must remain concurrent with LIGO's planned integration and alignment activities.

\section{1 "Clean as you go"}

A critical lesson learned at LIGO, and reiterated by $\mathrm{NIF}^{12}$, was the absolute necessity of a "clean as you go" philosophy. Even under ideal conditions, it is completely unrealistic not to anticipate some amount of contamination during months of activity near installed optics. The intricacy of the LIGO interferometer system further exacerbates the inevitable contamination control challenge because there are so many potential places for dust to hide, and those places become increasingly difficult to see and reach, as Figure 11 and Figure 12 demonstrate. In 2013, LIGO underwent a paradigm shift, and incorporated the cleaning on the go philosophy - regular cleaning following normal assembly work, before and after storage or transportation, before and after installation, and prior to closing a chamber.

High intensity flashlight arrays used at grazing incident angle on the optics and surrounding components significantly improve the ability to identify particulates, especially inside the dim chambers. $35 \mathrm{~W}$ and $3 \mathrm{~W}$ UV-A black lights ${ }^{\S}$ are also used to inspect non-optical surfaces, particularly during assembly. Figure 14 shows the debris accumulation after two days of assembly work in an ISO 5 (Class 100) cleanroom as viewed with a flashlight array vs. UV light. The bottom half of the part was dry-wiped, and the top half was not. The figure also demonstrates the UV flashlights' ability to highlight grease stains that might have been overlooked.

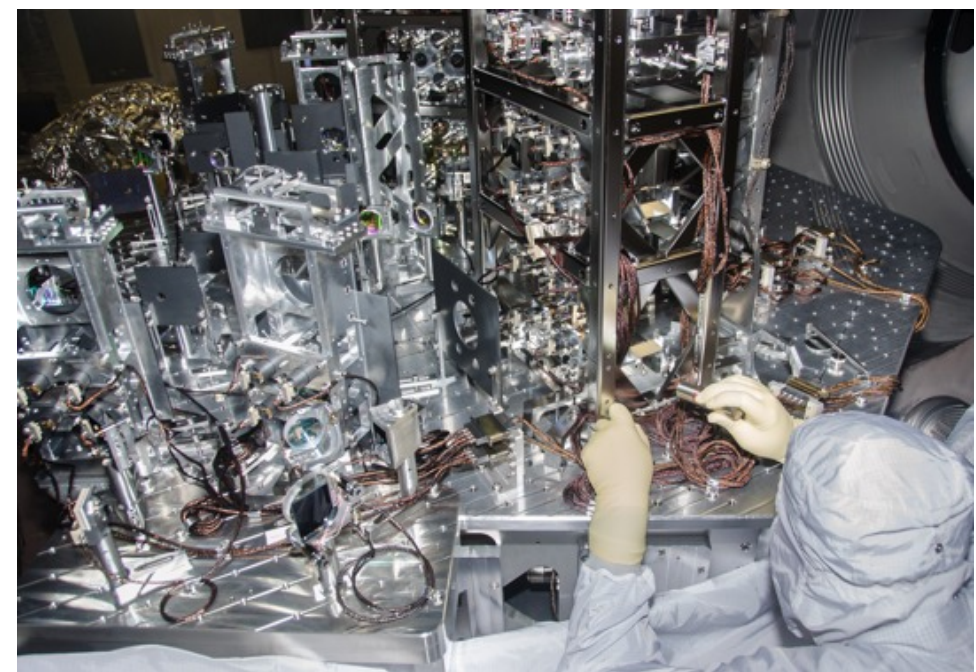

Figure 11. Integration and testing work in the crowded Livingston HAM2 chamber.

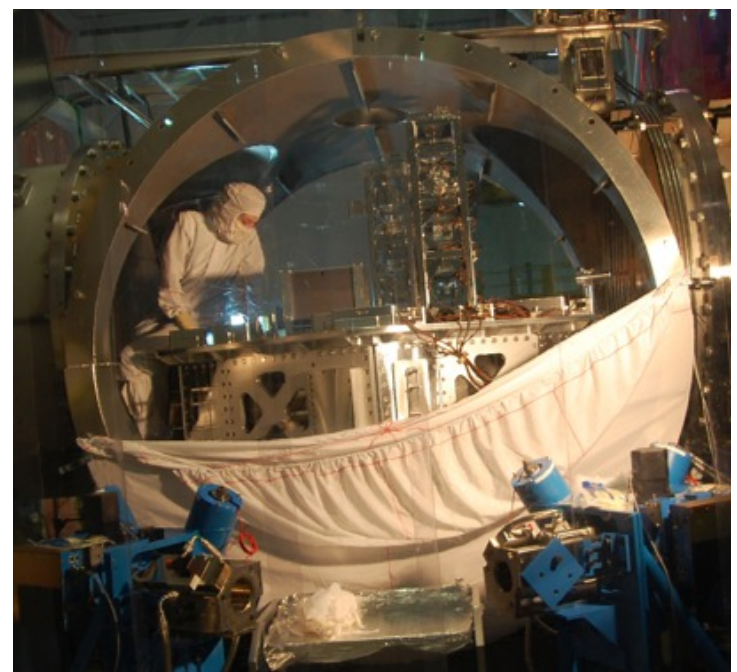

Figure 12. Maneuvering into HAM3 chamber for inspection of optical surfaces.

\footnotetext{
${ }^{\S}$ UV Light Technology Limited, P/N UV35WTORCH, www.uv-light.co.uk, +44 (0)121-423-2000
} 


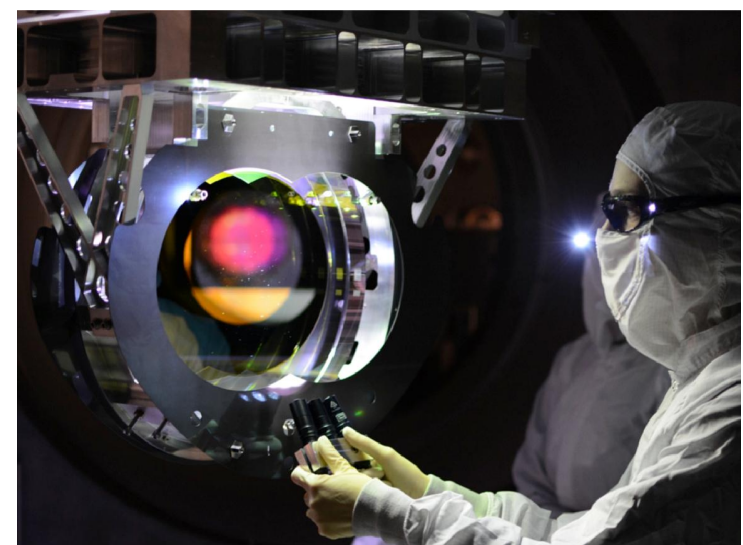

Figure 13. Inspecting the installed beam splitter optic for particulate contamination with a flashlight array.

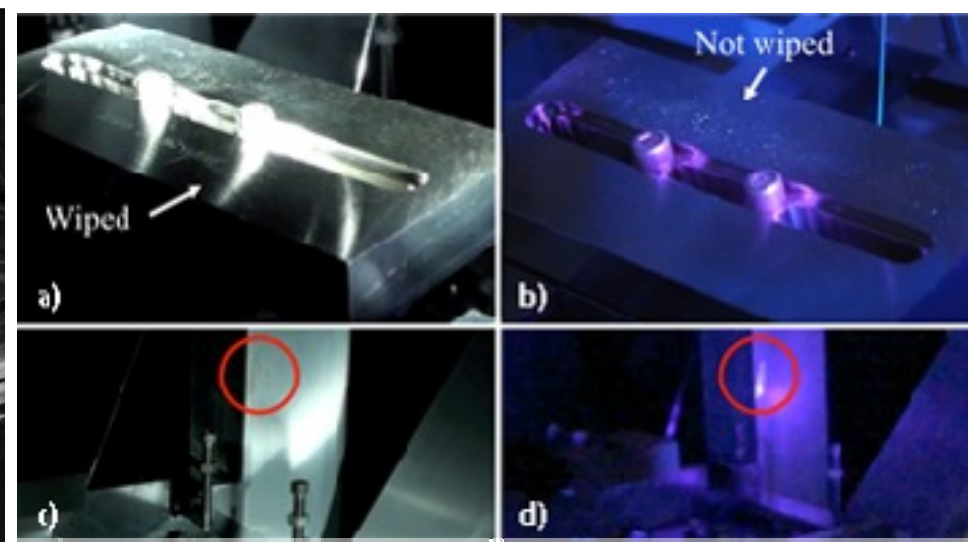

Figure 14. Inspection with a flashlight array (left) and UV light (right). The lower half of a Class A part was wiped clean in a) and b). Smudges appear is c) and d).

Custom Tiger-Vac vacuum cleaners ${ }^{* *}$, including backpack versions, facilitate frequent cleanup in assembly areas, decreasing the contamination added to the chambers during installation. Top Gun ${ }^{\mathrm{TM}}$ Ionizing Air Gun Systems ${ }^{\dagger \dagger}$, are used with Nitrogen to aid the cleaning of optics and to blow off debris on Class A and B mechanical surfaces.

Wiping with 99.5\% isopropanol (IPA) was promoted over dry wiping in chamber, and 50/50 IPA/water pre-saturated wipes are purchased and used in bulk for gross cleaning around vacuum chambers and assembly work areas. Instead of frequently changing cleanroom gloves, IPA pre-wetted wipes are now used to wash gloves immediately after donning a new pair, if they become contaminated with a small amount of particulates generated by cleanroom work, or if anything other than a Class A or B part is touched.

\subsection{Human factor}

People are a major source of contamination in cleanrooms. To better understand the contamination burden from people, it was important to evaluate LIGO's inventory of cleanroom garb, which is the current line of defense against human contamination. A series of physical exercises were performed inside an ISO 3 (Class 1) "Body Box" test chamber, where generated particles were collected at the knee and subfloor levels and reported at 1 minute intervals. Particle generation varies widely between individuals, so the same three test subjects (referred to here as Subject A, B, and C) participated in all the tests so relative comparisons could be drawn ${ }^{13}$. The variables for the trials were the age of the garb and the garments worn underneath the garb. Particle counts were averaged per subject during each new Body Box test for comparison.

To evaluate how well LIGO's garb (the exterior cleanroom suit) is holding up, shedding was considered, i.e., the particles generated by the garb itself. The subjects were given cleanroom scrubs, low particulating ESD micro denier uniforms, modeled by Subject A in Figure 17. The scrubs were worn under used cleanroom suits from 2008, 2010, and a brand new suit that had never been worn before. The result was the same for all three subjects, as there were only very minor differences in particle counts for the different ages of cleanroom suit. Counts for brand new suits are slightly higher, apparently the suits sheds a bit more initially then settle after a few washes. From these tests it was surmised that the older LIGO garb was performing comparably to newer garb.

Shielding, or particle penetration through the garb, was investigated using street clothes as a constant and varying the age of the garb. Each test subject wore personal clothing of his or her choice beneath 2010 and new garb. For the older 2010 garb, there was a noticeable increase in total particles shed for two of the three subjects. Newer garb appears to be a better barrier than older garb.

\footnotetext{
${ }^{* *}$ Tiger-Vac, P/N MV-1CR (BP) Series, www.tiger-vac.com, (800) 688-4437

${ }^{\dagger}$ Simco-Ion, Model TopGun ${ }^{\mathrm{TM}}$, www.simco-ion.com/industrial.aspx, (800) 203-3419
} 


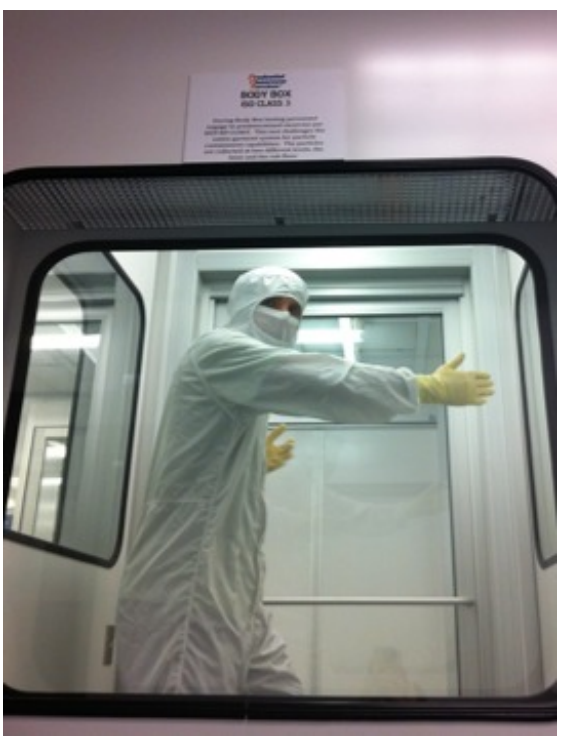

Figure 15. Subject A performing Body Box test exercises in old garb.
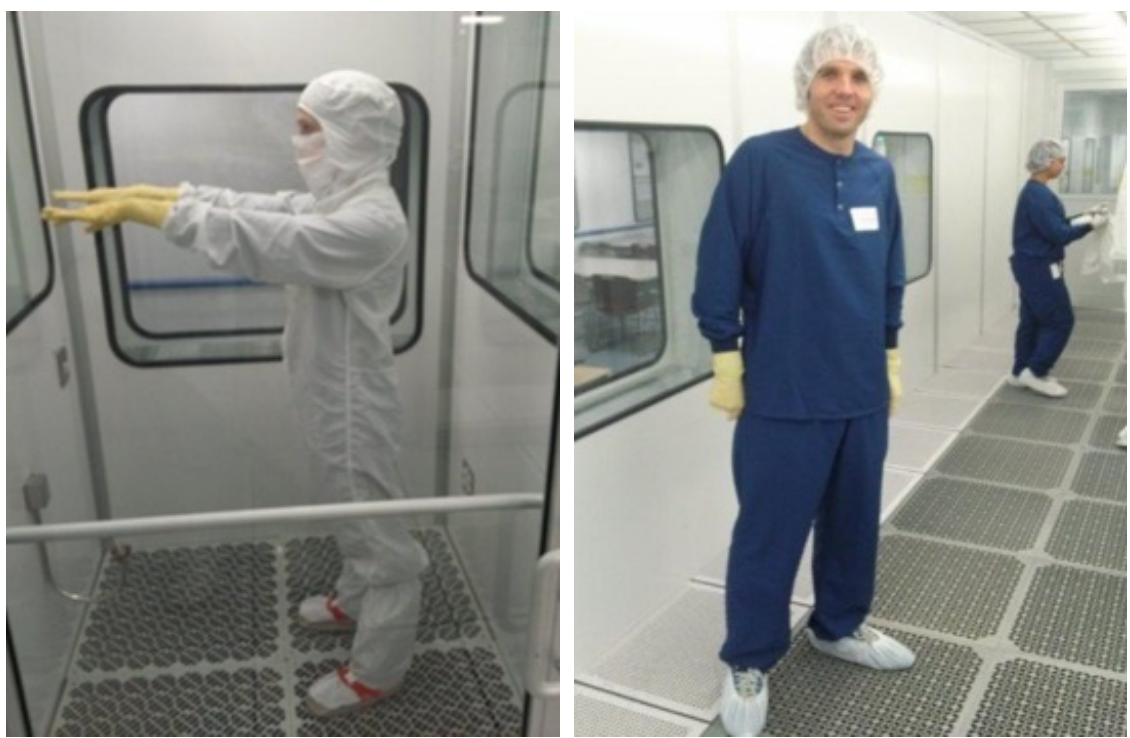

Figure 16. Subject $\mathrm{C}$ performing Body Box Figure 17. Subject A modelling micro test exercises in brand new garb. denier scrubs.

\section{Scrubs vs Street Clothes}

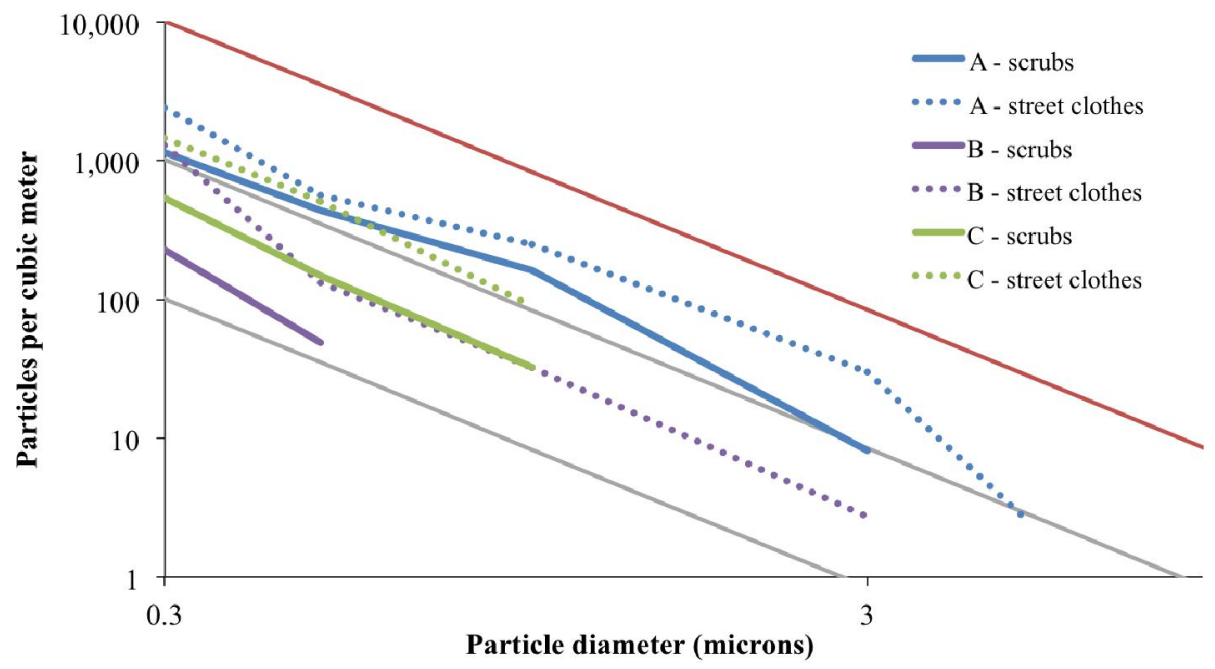

Figure 18. Graph of particles generated by 3 subjects during Body Box tests with scrubs and street clothes.

The most notable finding from these tests was the reduction of particles for all the subjects when wearing scrubs under the garb. For one subject, there was a one-half class improvement and for the remaining two subjects, there was an entire cleanroom class improvement (factor of 10 reduction) from wearing scrubs under the external cleanroom suit. As a result of these impressive improvements in particle count, scrubs have been implemented at both observatories.

\subsection{Facilities}

In addition to the cleaning and mitigation techniques described in this document the team has also worked at enhancing our basic cleanliness practices. For example switching to using double buckets for cleaning the floors, along with NovaClean ${ }^{\mathrm{TM}+\mathrm{clean} r o o m}$ floor cleaner has made a notable difference to contamination levels.

\footnotetext{
$\$$ Micronova Manufacturing Inc., P/N NC1-Q, www.micronova-mfg.com, (888) 816-4276
} 


\section{CLEANING AND PROTECTING OPTICS IN-SITU}

By employing all the techniques above, the contamination on optical surfaces can be minimized. However, it is impossible to keep optics completely clean through the 3-5 months of necessary installation work each chamber sees when opened. Thus, there had to be a plan for restoring optics to a clean state after they had been hung in suspensions and installed into chambers. This plan would ideally not involve mechanical stress that might scratch delicate coatings, or add too much extra time to the install schedule. It also cannot involve removing the optics from their suspensions, as that is very difficult once they are in place. That leaves finding a way of cleaning suspended optics inside a cramped vacuum chamber, quickly, and leaving a minimum of hydrocarbon and particulate contaminants from the process in the vacuum chamber upon exit.

The cleaning method that was researched as a solution to this problem uses a cleaning polymer called First Contact ${ }^{\mathrm{TM}}$ $(\mathrm{FC})^{\S \S}$. FC is a mixture of solvent and polymer that is applied in liquid form to an optical surface, let dry, and then removed to leave a clean optic. LIGO subjected FC to a significant round of internal testing before qualifying it for use.

\subsection{Testing and qualification}

One important test was to the possibility that FC left residue behind on optics after removal, hydrocarbons being the biggest concern. Pristine glass blanks were swabbed with Freon, along with optics that had been cleaned with First Contact $^{\mathrm{TM}}$. The Freon was then put through an FTIR scan. The results of these tests found no measurable difference between optics that had been cleaned with FC and pristine glass blanks. In fact this was tested on two occasions, in $2006^{14}$ and once again in $2010^{15}$.

FC was qualified for use in the vacuum chamber by leaving several samples in a small vacuum chamber for a few months, and then monitoring their outgassing with an RGA scans ${ }^{16}$.

\subsection{Cleaning efficiency}

A series of scatter measurements using a Bidirectional Reflectance Distribution Function (BRDF) scatterometer tracked the cleaning performance of FC on dielectric coated optics. FC was tested both by itself with this method, as well as against other typical methods of cleaning optics, for example methanol drag wiping. The full list of these tests has been recorded in internal document ${ }^{17}$.

A highlight of the BRDF tests shows that repeated applications of FC only improves optical surfaces ${ }^{17}$, see Figure 19 below. Note that these tests were done in 2010, and the LIGO application and removal procedures have only improved
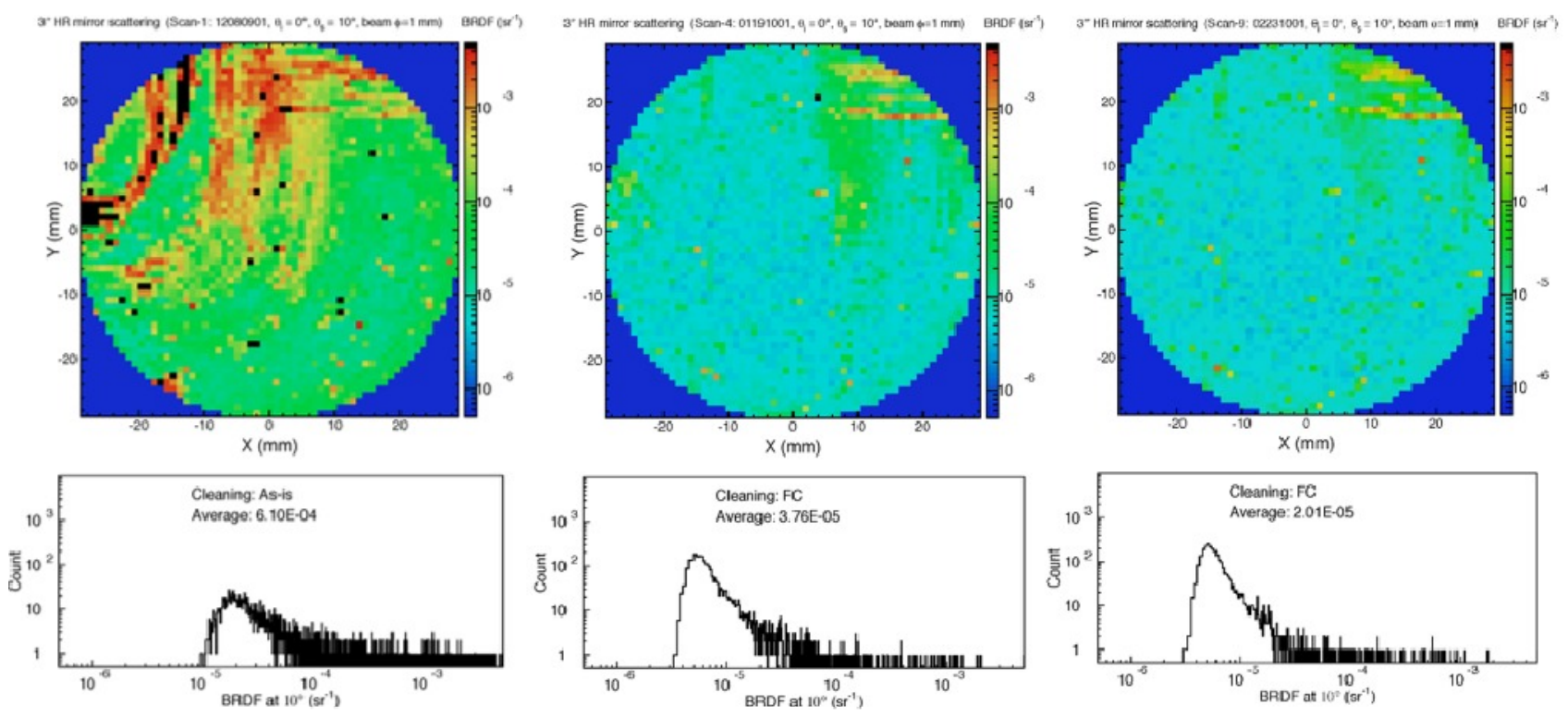

Figure 19. Progression of BRDG scans as optic is cleaned with First Contact ${ }^{\mathrm{TM}}$.

\footnotetext{
$\S \S$ Photonic Cleaning Technologies, P/N RFCF and RTFCF, www.photoniccleaning.com, (888) 308-6116
} 


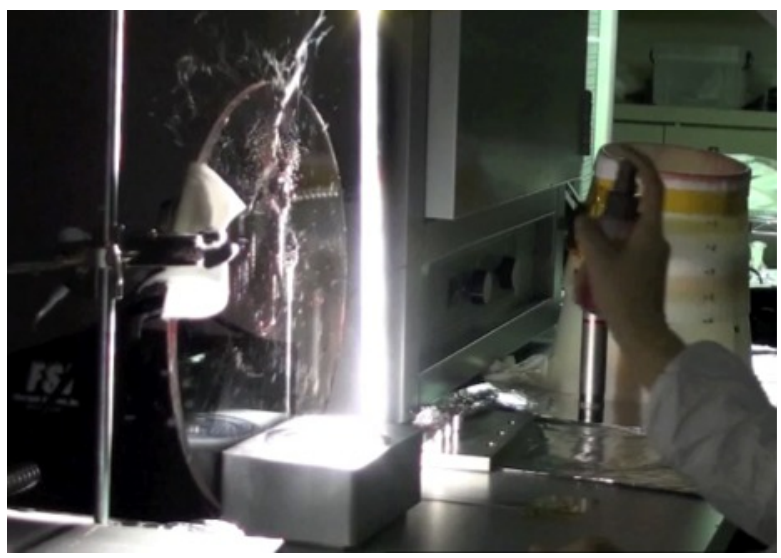

Figure 20. Polymer cobwebs created by wrong polymer/solvent mix.

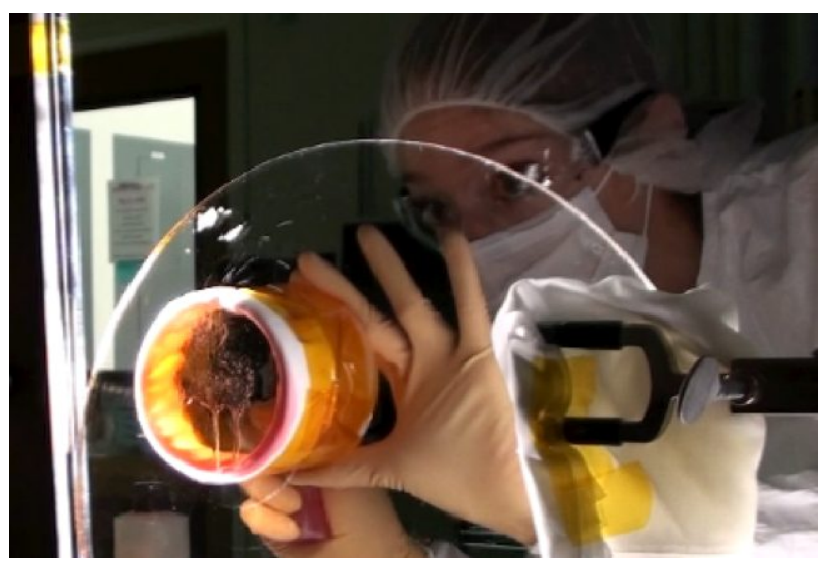

Figure 21. Testing a prototype protective cone.

since then.

\subsection{Non-contact application techniques}

Once First Contact ${ }^{\mathrm{TM}}$ was approved for use at LIGO, research began on how to successfully apply and remove it. There were concerns that the mechanical action of brushing it on may drag contaminates across the surface hard enough to scratch a coating. This approach is not encouraged for delicate coatings. This led LIGO towards non-contact application approaches by either pouring or spraying.

Experiments with spray application were made with a variety of techniques; there were many failed approaches because of the difficulty of using a spray nozzle to apply solvent and polymer with any degree of precision. Also, it took a while to get the mix of polymer to solvent right: if there is too much polymer in solution then it will dry almost the instant it comes out of the spray nozzle, creating cobwebs of polymer that can contaminate the chamber around the optic that is being cleaned. Figure 20 shows an example of this in action. Once the right mix for spraying was determined, a device for protecting the rest of chamber was tested. Figure 21 shows testing of a prototype of this protective "spray cone".

The optimal spray mix and bottle was found to be a $3 \mathrm{oz}$ HDPE bottle and atomizing nozzle, filled with a 1:1 ratio of red First Contact ${ }^{\mathrm{TM}}$ : red FC thinner. This mix lowers the polymer content enough to avoid cobwebs. When coupled with good technique and use of the protective cones, back splatter in chamber is avoided.

Having determined that FC was acceptable to use to clean LIGO optics, and establishing how to apply it without creating cobwebs or residue, that left the challenge of access to optical surfaces in chamber.

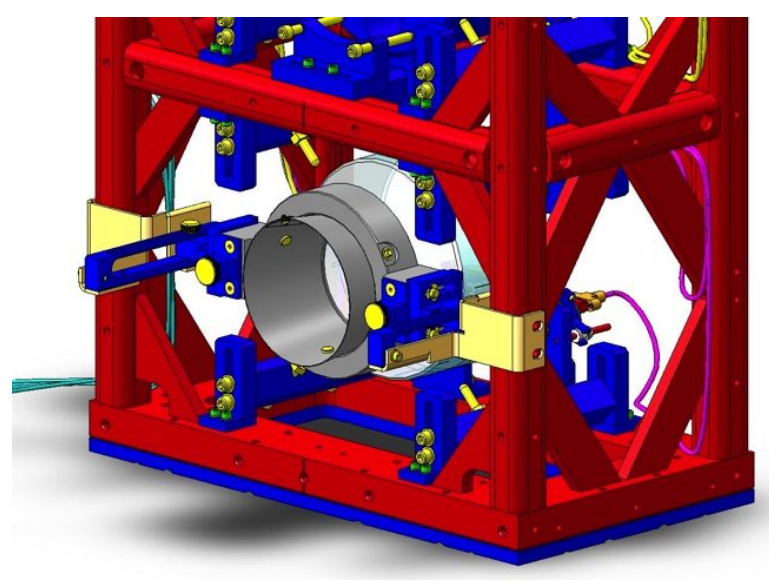

Figure 22. Design of protective cone bolted to a suspension. Figure 23. Final product at work.

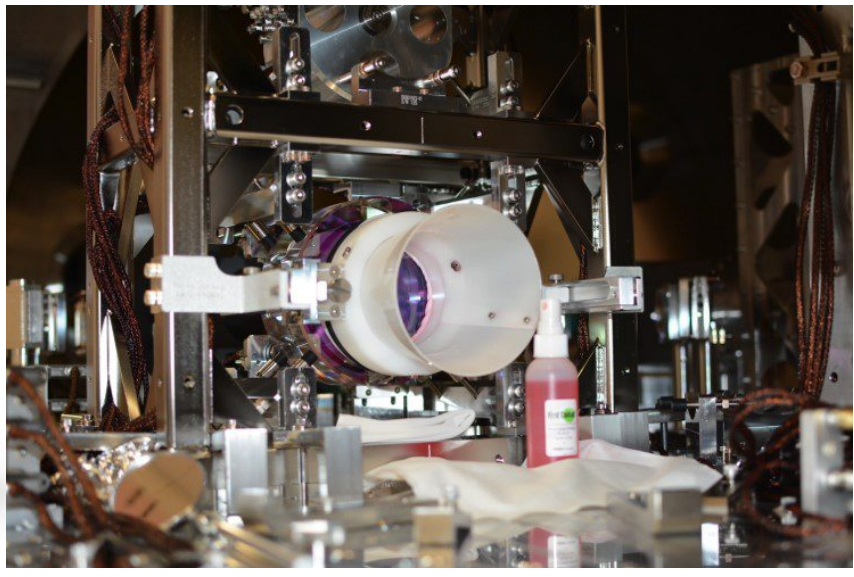


Figure 11 and Figure 12 emphasize the logistical difficulties involved with accessing components in chamber, making spraying a polymer onto an optic even more challenging. The design of the protective cones solved this problem. The protective shields make a seal around the outer diameter of the optical surface using cleanroom wipes where it actually contacts, to prevent polymer drips from spray. Also, they bolt straight to the suspension, leaving hands free to apply the cleaning polymer, and they are a small enough footprint to avoid hitting any of the other non-removable optics and equipment that are on the table.

As a result of these tests, a successful "no-contact" cleaning approach for delicate optical coatings has been created, using the method for precision application of cleaning polymer to optics inside a vacuum chamber that was described above, and with a protective device to shield the rest of the chamber from contamination as shown on the in Figure 22. Figure 23 is a demonstration of the final product in action, after cleaning suspended optics inside a LIGO HAM vacuum chamber at the LIGO Livingston Observatory.

\section{CONCLUSIONS}

Over the past 1.5 years LIGO has accrued a variety of powerful tools and techniques to manage contamination in its high-vacuum systems, through internal research and development as well as collaboration with external industry contacts, such as NIF.

We are continuing to develop further techniques including a method of cleaning optics in-situ using a modified air knife or air nozzle system (similar to that in use by NIF). In addition we are looking at provision to enhancing our facilities on site by utilizing cross-flow HEPA filters and custom flooring close to our chambers.

As a result of these ongoing efforts, we now have a better understanding of the contamination levels and sources and have made significant improvements to methods of controlling contamination and protecting the optics from damage in the Advanced LIGO Interferometers.

\section{ACKNOWLEDGEMENTS}

The authors gratefully acknowledge the support of the United States National Science Foundation (award No. PHY-07 57058). LIGO was constructed by the California Institute of Technology and Massachusetts Institute of Technology with funding from the National Science Foundation, and operates under cooperative agreement PHY-0107417. Caltech also acknowledges financial support from the Science and Technology Facilities Council of the United Kingdom. This paper filed internally under LIGO document number LIGO-P1300192. LIGO Internal Reports may be accessed at https://dcc.ligo.org/cgi-bin/DocDB/DocumentDatabase.

The authors wish to acknowledge the many colleagues in LIGO and the LIGO Scientific Collaboration who have supported this work. In particular, the authors are grateful for the guidance and support of Norna Robertson, Eric Gustafson, Garilynn Billingsley, and Dennis Coyne of LIGO Engineering at Caltech. The work would not have been possible without the outstanding efforts and collaboration of the installation teams at the LIGO observatories, particularly Matt Heintze at Livingston and Betsy Weaver at Hanford. In addition, the authors would like to thank industry contacts from the National Ignition Facility and Prudential Cleanroom Services, for their guidance and open collaboration. 


\section{REFERENCES}

[1] Harry, G. M., “Advanced LIGO: The Next Generation of GW Detectors," Class. Quantum Grav. 27(8), 084006 (2010).

[2] Coyne, D., Some Thoughts Regarding Particulate Contamination Control Requirements, LIGO-T1300511-v11, June 5, 2013.

[3] Bland, B., Fauver, J., Heintze, M., Torrie, C., Coyne, D., aLIGO Contamination Control Plan, LIGO-E0900047v21, February 20, 2013.

[4] Coyne, D., LIGO Vacuum Compatible Materials List, LIGO-E960050-v12, May 7, 2013.

[5] Waterbury, S., "Outgassing Data for Selecting Spacecraft Materials Online," NASA Outgassing, 26 June 2013, http://outgassing.nasa.gov/ (1 October 2013).

[6] Bland, B., Coyne, D., Fauver, J., LIGO Clean and Bake Methods and Procedures, LIGO-E960022-v25, July, 3, 2012.

[7] Stowers, IF IEST-STD-1246D Product Cleanliness Levels and Contamination Control Program. Institute of Environmental Sciences and Technology, 940 E. Northwest Highway, MT. Prospect, IL 60056.

[8] Stowers, IF., and Ravizza, DL., The Particle Cleanliness Validation System, Proceedings 48th Annual Technical Meeting of the Institute of Environmental Science and Technology, April 28-May 1, 2002, UCRL-JC145932

[9] Gushwa, K. The LIGO Particle Cleanliness Validation System LIGO-T1300665, October $27^{\text {th }} 2013$.

[10] AmScope ToupView for Windows, copyright 203-20012, Version 3.7.939.

[11] Coyne, D. and Taylor, B., LIGO-E1300015-v1: aLIGO FTIR Sample Record Test Dust Sample using Forensic sampling kits. March 2013 LIGO Internal Report. March $28^{\text {th }} 2013$.

[12] Pryatel, J., and Gourdin, W., Clean Assembly Practices to Prevent Contamination and Damage to Optics, Boulder Damage Symposium, January 5, 2006.

[13] Gushwa, K. Torrie, C., Phelps, M., LIGO-T1300093, Prudential BodyBox Results. Novemebr $1^{\text {st }} 2013$. LIGO Internal Report.

[14] Armandula, H., Markosyan, A., and Anderson, M., LIGO-T060161-x0 FTIR Test Of First ContactTM, July 2006. LIGO Internal Report.

[15] Phelps, M., LIGO-E1000128-v1 FTIR: First Contact and peek mesh May 2010. LIGO Internal Report.

[16] Coyne, D., LIGO-T0900438-v2 LIGO Vacuum Qualification of First Contact June 2011. LIGO Internal Report.

[17] Phelps, M., LIGO-T1000137-v3, Drag Wiping and First Contact BRDF Tests. LIGO Internal Report. 